University of Wollongong

Research Online

Faculty of Engineering and Information

Faculty of Engineering and Information

Sciences - Papers: Part A

Sciences

$1-1-2014$

\title{
Economic-emission dispatch problem: A semi-definite programming approach
}

A M. Jubril

Obafemi Awolowo University

O A. Olaniyan

Obafemi Awolowo University

O A. Komolafe

Obafemi Awolowo University

Philip O. Ogunbona

University of Wollongong, philipo@uow.edu.au

Follow this and additional works at: https://ro.uow.edu.au/eispapers

Part of the Engineering Commons, and the Science and Technology Studies Commons

Research Online is the open access institutional repository for the University of Wollongong. For further information contact the UOW Library: research-pubs@uow.edu.au 


\title{
Economic-emission dispatch problem: A semi-definite programming approach
}

\author{
Abstract \\ A semi-definite programming (SDP) formulation of the multi-objective economic-emission dispatch \\ problem is presented. The fuel cost and emission functions are represented by high order polynomial \\ functions and this was shown to be a more accurate representation of the economic-emission dispatch \\ (EED) problem. Furthermore, the polynomial functions of both objective functions are aggregated into a \\ single objective function using the weighted sum approach. This thus reduces the problem to a standard \\ polynomial optimization problem which was formulated as a hierarchy of semi-definite relaxation \\ problems. The resulting SDP problem was then solved at different degrees of approximation. The \\ performance of the proposed approach was evaluated by conducting experiments on the standard 6-unit \\ and the 13-unit IEEE test systems. The results obtained were compared with those reported in the \\ literature and indicated that SDP has inherently good convergence property and provides better \\ exploration of the Pareto front. \\ Keywords \\ economic, problem, approach, dispatch, emission, programming, definite, semi \\ Disciplines \\ Engineering | Science and Technology Studies \\ Publication Details \\ Jubril, A. M., Olaniyan, O. A., Komolafe, O. A. \& Ogunbona, P. O. (2014). Economic-emission dispatch \\ problem: A semi-definite programming approach. Applied Energy, 134 (December), 446-455.
}




\title{
Economic-Emission Dispatch Problem: A Semi-definite Programming Approach
}

\author{
A. M. Jubril ${ }^{\mathrm{a}, *}$, O. A. Olaniyan ${ }^{\mathrm{a}}$, O. A. Komolafe ${ }^{\mathrm{a}}$, P. O. Ogunbona ${ }^{\mathrm{b}}$ \\ ${ }^{a}$ Department of Electronic and Electrical Engineering, Obafemi Awolowo \\ University,Ile-Ife, Nigeria \\ ${ }^{b}$ School of Computer Science and Software Engineering, University of Wollongong, \\ Australia
}

\begin{abstract}
A semi-definite programming (SDP) formulation of the multi-objective economic-emission dispatch problem is presented. The fuel cost and emission functions are represented by high order polynomial functions and this was shown to be a more accurate representation of the economic-emission dispatch (EED) problem. Furthermore, the polynomial functions of both objective functions are aggregated into a single objective function using the weighted sum approach. This thus reduces the problem to a standard polynomial optimization problem which was formulated as a hierarchy of semidefinite relaxation problems. The resulting SDP problem was then solved at different degrees of approximation. The performance of the proposed approach was evaluated by conducting experiments on the standard 6-unit and the 13-unit IEEE test systems. The results obtained were compared with those reported in the literature and indicated that SDP has inherently good convergence property and provides better exploration of the Pareto front.
\end{abstract} Keywords:

multi-objective optimization, polynomial optimization, economic dispatch,

\footnotetext{
${ }^{*}$ Corresponding Author: A. M. Jubril, ajubril@oauife.edu.ng; Tel: +234 8056568457 Preprint submitted to Applied Energy 
emission dispatch, semidefinite programming, weighted sum method

\section{Introduction}

There has been an increased awareness of the environmental impact of the gaseous emissions from fossil-based electricity generating plants operated by utilities in various countries. The pace of economic development in many developing countries has placed high demand on the generation of electrical energy to drive emerging industries. Thus, the response to the challenge posed by the emissions has been, in some cases, tighter regulations on the operation of generating plants. More specifically, utilities are being requested to modify the strategy of economic dispatching of generating plants to incorporate environmental constraints. Reduction in atmospheric pollution and emission has therefore become significant objective in the electrical power dispatch problem. For example, in [1] the impact of the US Clean Air Act of 1990 on the economic dispatch problem was considered. Several strategies including the use of low emission fuels, replacement of the aged fuel-burners with cleaner ones, use of natural gas and emission dispatching have been suggested $[1,2,3]$. In general, the strategies developed to address emission reduction and control problems can be broadly divided into two groups [4] namely: emission methods and minimum cost methods. The minimum emission methods are aimed at minimizing the total emission [5] or the distributed emission function, that is based on the emission dispersion model. The dispersion model provides emission limits for different geographical locations [6]. It has been noted that this method can be inaccurate and is computationally intensive [4]. Minimum cost methods integrate the emission objective 
into the problem as a constraint. Some of the costs that have been considered in single and multi-objective cases include fuel cost, emission taxes, and emission worth $[7,8]$. Perhaps the most attractive option was the combined emission-economic dispatch (EED) [9] in which the problem is formulated as a multi-objective optimization whose solution underpins the operating strategy. This has led to renewed interest by the research community and the utilities to address the dispatch problem as a multi-objective optimization task involving both generating cost and environmental constraints.

In general, multi-objective optimization problem consists of a number of objectives to be optimized simultaneously and is associated with a number of equality and inequality constraints. It can be written as

$$
\begin{aligned}
& \underset{\mathbf{x} \in X}{\operatorname{minimize}}\left[f_{1}(\mathbf{x}), f_{2}(\mathbf{x}), \ldots, f_{p}(\mathbf{x})\right] \\
& \text { where } \\
& X=\left\{\mathbf{x} \in \mathbf{R}^{n}: g(\mathbf{x}) \leq 0, h(\mathbf{x})=0\right\}
\end{aligned}
$$

In (1) $X$ is the feasible region in the decision space; $f_{i}(\mathbf{x})$ is the $i^{\text {th }}$ objective function; $\mathbf{x}$ is a decision vector; $p$ is the number of the objectives; $g(\mathbf{x})$ and $h(\mathbf{x})$ are the inequality and equality constraints respectively. In single objective optimization problem, the optimal solution is that value $\mathbf{x}^{*} \in X$ such that no other value $\mathrm{x} \in X$ gives a better objective value. However in multi-objective optimization problem, there is no unique optimal solution, rather there is a set of optimal solutions which are non-inferior to any other in the set.

A vector $\mathbf{u}=\left[u_{1}, \ldots, u_{p}\right]$ is said to dominate another vector $\mathbf{v}=\left[v_{1}, \ldots, v_{p}\right]$ (denoted by $\mathbf{u} \preceq \mathbf{v}$ ) if and only if $\mathbf{u}$ is partially less than $\mathbf{v}$; that is

$$
\forall i \in 1, \ldots, p, u_{i} \leq v_{i} \wedge \exists i \in 1, \ldots, p: u_{i}<v_{i} .
$$


A solution $\mathrm{x}^{*} \in X$ is said to be Pareto optimal if and only if there is no $\mathbf{x} \in X$ for which $\mathbf{F}\left(\mathbf{x}^{*}\right)=\left(f_{1}\left(\mathbf{x}^{*}\right), \ldots, f_{p}\left(\mathbf{x}^{*}\right)\right)$ dominates (or is better than) $\mathbf{F}(\mathbf{x})=\left(f_{1}(\mathbf{x}), \ldots, f_{p}(\mathbf{x})\right)$.

Methods of evaluating multi-objective algorithms include consideration for the closeness of the generated solution set to the ideal Pareto front; this is termed convergence of the solution set. In addition, the uniformity and spread of the generated solution, termed the diversity, are are also quantified [10]. Although generated solution sets of multi-objective optimization problems are required to satisfy both convergence and diversity criteria, the two criteria are rarely well satisfied in any one algorithm.

The multi-objective EED problem is usually formulated as in (1) and we will provide details later in the paper. In the next section we provide a review of the main approaches to multi-objective EED problem.

\section{Related Work}

Several methods have been proposed to solve the multi-objective EED problem. In general, the multi-objective optimization problem is converted into a single objective problem. In [11], the weighted sum method was considered. This method generates non-dominated solutions by varying the weight factor [12]. However, it is unable to capture non-convex part of the trade-off surface. Attempts to produce a uniform distribution of the solution points on the trade-off surface through an even variation of the weight factor has not been successful. The $\varepsilon$-constrained method overcomes the problem of finding points on the non-convex part of the trade-off surface. However, the method incurs high computational burden and tends to find weakly 
dominated points [13]. More recent approaches use evolutionary algorithms $[14,15]$ including particle swarm optimization (PSO) method $[16,17]$, genetic algorithms (GA) [9], bacterial foraging (BF) method [18, 19] and differential evolution [20]. Although evolutionary algorithms can be efficient, they are computationally expensive and may result in premature convergence.

In [21], a linear programming (LP) method was proposed to efficiently handle inequalities and detection of infeasible solutions. However, all the nonlinear constraints and objective functions need to be linearized about the operating point. This linearization step is not required in a semi-definite programming (SDP) formulation which is a generalization of the LP technique. Most multi-objective evolutionary algorithms are population-based and can generate the Pareto front estimate in a single run. However due to their stochastic nature, the attainment of the optimal solution set may be difficult or even impossible. In contrast, the SDP method is not populationbased and only require several runs to generate an estimate of the Pareto set. The fact that the SDP formulation is convex ensures that it will provide globally optimum Pareto solutions.Perhaps a most important advantage of SDP problems is that they can be efficiently solved. Other features include the ease of reformulating various other nonlinear problems as SDP, and the availability of high-quality solvers e.g. SeDuMi, SPDT3, etc [22]. There are also recent results that have extended most interior point methods for LP to solve the SDP [23, 24].

While most optimization problems in power systems have polynomial objective functions and constraints, very few applications of SDP have been reported in power system literature [25]. Examples of applications of SDP 
are those presented in $[25,26,27,28,29]$ and [30]. We note that these applications have been limited to economic dispatch (ED) problems with quadratic objective and constraint functions. The use of second order fuel cost function in dispatch problems only gives a rough approximation of the generator cost function and the corresponding solution deviates from the true value. In $[31,32]$ higher order polynomial cost function has been used to obtain an improved solution to ED problem. Specifically, the work reported in [31] considered cubic function models of fuel cost and the emission functions. In this paper we examine the use of higher degree polynomial in approximating the exact objective function for the EED problem and the effectiveness of the SDP method in solving the resulting polynomial problem. Additionally, we provide comparative evaluation against results reported in the literature.

This paper is motivated by the recent advances in the solution of polynomial optimization problem (POP) using the semi-definite program. Although polynomial optimization problems are generally non-convex and difficult to solve, various hierarchy of convex relaxation of the problem have been proposed which monotonically converge to the exact global optimal solution $[33,34,22]$. The relaxation allows non-convex problems to be solved by convex optimization techniques. Also, unlike most multi-objective evolutionary algorithms (MOEA) which are stochastic optimizers and which find it difficult or even impossible to attain the ideal Pareto surface, SDP provides a cheaply computable lower bound of the minimum value [23]. Therefore, it has the good property of providing convergence to solution set that are close to the ideal Pareto surface.

To the best of our knowledge, SDP has not been applied to solve the EED 
problem. Our contribution thus extends the SDP application to the EED problem and ED problems with polynomial functions. The EED problem is formulated as a polynomial optimization problem in a finite dimensional space and solved as a semi-definite program. Polynomial optimization problems are generally non-convex and SDP relaxation of non-convex problems has been applied to transform the problem into a convex approximation. Application of the weighted sum to the resulting convex problem guarantees the capture of the entire Pareto front. This solution strategy extends the application of the weighted sum method which has been limited due to its inability to capture solution points on non-convex part of the Pareto front.

We organize the rest of the paper as follows. In Section 3 we provide the formulation of the economic-emission dispatch problem, highlighting generation cost as well as the polynomial expression for the pollutant emissions. These are then combined in multi-objective optimization problem. The thrust of this paper is the use of SDP in solving multi-objective EED problem. In Section 4 the semi-definite program is formulated and a sketch of the polynomial approximation problem along with its SDP relaxation is provided. We consider the polynomial approximation of the exponential term appearing in the emission function in Section 5. This is followed by a formulation of the EED as polynomial optimization problem. We discuss the solution of the resulting problem using MATLAB software - SparsePOP and SeDuMi. Evaluation of the results of our proposed approach based on some case studies are provided in Section 6. The IEEE test systems with 6 and 13 generating units were used to set up case studies considering results with and without transmission losses in the power balance equation. We draw 
conclusions in Section 7.

\section{Economic-emission Dispatch Problem Formulation}

The development of the economic-emission dispatch problem considers the objectives involving generator fuel costs and the pollutant emission. The emission gasses of interest are the sulphur $\left(\mathrm{SO}_{x}\right)$, carbon $\left(\mathrm{CO}_{2}\right)$ and nitrogen $\left(N O_{x}\right)$ oxides. Usually, the constraints are related to the generation capacities and power balance of the plants.

\subsection{Problem Objectives}

\subsubsection{Total Fuel Cost, $C\left(P_{g}\right)$}

The generator costs are generally represented by quadratic functions. The total fuel cost, $C\left(\mathbf{P}_{\mathbf{g}}\right)$, can then be expressed as

$$
C\left(\mathbf{P}_{\mathbf{g}}\right)=\sum_{i=1}^{p} a_{i}+b_{i} P_{g_{i}}+c_{i} P_{g_{i}}^{2},
$$

where $C$ is the total fuel cost, $P_{g_{i}}$ is the real power output of the $i^{\text {th }}$ plant, and $a_{i}, b_{i}$, and $c_{i}$ are the fuel cost coefficients of the plant. A more general expression for the total fuel cost is a polynomial function of degree $k$,

$$
C\left(\mathbf{P}_{\mathbf{g}}\right)=\sum_{i=1}^{p} \sum_{j=0}^{k} \alpha_{i j} P_{g_{i}^{j}},
$$

where $\alpha_{i j}$ are the corresponding the fuel cost coefficients of the plant. This allows other features such as the valve-point loading effect [35] and the piecewise linear property of the combined cycled co-generation plants, of the fuel cost to be captured in the representation [36]. 


\subsubsection{Pollutant Emission}

It has been observed that the $S O_{x}$ emissions, $E_{S O_{x}}$, are proportional to the fuel consumption of the thermal unit and, therefore takes the same form as the fuel cost. Thus,

$$
E_{S O_{x}}\left(\mathbf{P}_{\mathbf{g}}\right)=\sum_{i=1}^{p} \alpha_{i}+\beta_{i} P_{g_{i}}+\gamma_{i} P_{g_{i}}^{2} .
$$

Carbon dioxide $\left(\mathrm{CO}_{2}\right)$ emission poses a global warming problem, and is considered as one of the important environmental pollutants. The $\mathrm{CO}_{2}$ emission function, $E_{\mathrm{CO}_{2}}$, can be approximated by a linear equation of the unit's power output as [37]:

$$
E_{C O_{2}}\left(\mathbf{P}_{\mathbf{g}}\right)=\sum_{i=1}^{p} \tau_{i} P_{g_{i}}
$$

where $\tau_{i}$ are $\mathrm{CO}_{2}$ emission coefficients.

However, the $N O_{x}$ emission, $E_{N O_{x}}$, is a highly non-linear function of the generated power. This was assumed to be a combination of a polynomial and exponential functions of the generated power [5]. An emission function of the form

$$
E_{N O_{x}}\left(\mathbf{P}_{\mathbf{g}}\right)=\sum_{i=1}^{p} \alpha_{i}+\beta_{i} P_{g_{i}}+\zeta_{i} \exp \left(\lambda_{i} P_{g_{i}}\right),
$$

was implemented in [5]. The second order polynomial form is widely used in the literature [2], but the form in (7) has been observed to provide more accurate representation [5]. Combining the $\mathrm{SO}_{x}, \mathrm{CO}_{2}$ and $\mathrm{NO}_{x}$ emission of all the units, the total emission of the atmospheric pollutants $\left(\mathrm{SO}_{x}, \mathrm{CO}_{2}\right.$, $\left.N O_{x}\right)$ in ton/h can be expressed as

$$
E\left(\mathbf{P}_{\mathbf{g}}\right)=\sum_{i=1}^{p}\left(\alpha_{i}+\beta_{i} P_{g_{i}}+\gamma_{i} P_{g_{i}}^{2}\right)+\zeta_{i} \exp \left(\lambda_{i} P_{g_{i}}\right),
$$


where $\alpha_{i}, \beta_{i}, \gamma_{i}, \lambda_{i}$ and $\zeta_{i}$ are the coefficients of the $i^{\text {th }}$ plant emission characteristics. In some cases, the emission function is approximated by a higher order of polynomial. An example is the cubic polynomial function expressed as

$$
E\left(\mathbf{P}_{\mathbf{g}}\right)=\sum_{i=1}^{p} \alpha_{i}+\beta_{i} P_{g_{i}}+\gamma_{i} P_{g_{i}}^{2}+\delta_{i} P_{g_{i}}^{3} .
$$

\subsection{Problem Constraints}

\subsubsection{Generation capacity constraints}

The real power output of each generating unit is constrained between the upper and lower limits as follows:

$$
P_{g_{i}}^{\min } \leq P_{g_{i}} \leq P_{g_{i}}^{\max }, \quad i, \ldots, p
$$

This defines the inequality constraint $g\left(\mathbf{P}_{\mathbf{g}}\right)$.

\subsubsection{Power balance constraint}

The power balance constraint is given by

$$
\sum_{i=1}^{N} P_{g_{i}}=P_{D}+P_{L}\left(\mathbf{P}_{\mathbf{g}}\right)
$$

where $P_{D}$ is the total load demand, and $P_{L}\left(\mathbf{P}_{\mathbf{g}}\right)$ is the transmission loss. Using the Kron's loss formula, $P_{L}\left(\mathbf{P}_{\mathbf{g}}\right)$ can be expressed as

$$
P_{L}\left(\mathbf{P}_{\mathbf{g}}\right)=\sum_{i=1}^{p} \sum_{j=1}^{p} P_{g_{i}} B_{i j} P_{g_{j}}+\sum_{i=1}^{p} B_{i 01} P_{g_{i}}+B_{00}
$$

where $B_{i j}, B_{i 01}$ and $B_{00}$ are the Kron's loss coefficients. Equation (11) defines the equality constraint $h\left(\mathbf{P}_{\mathbf{g}}\right)$. 


\subsubsection{Spinning reserve constraints}

The available spinning reserve, $R_{i}$, of the $i^{\text {th }}$ generating unit depends on its maximum output power. This is constrained as follows:

$$
R_{i} \leq P_{i_{\max }}-P_{g_{i}}
$$

The total spinning reserve of the whole system is finite and equal to a specified value $P_{S R}$, expressed as

$$
\sum_{i=1}^{n_{g}} R_{i}=P_{S R}
$$

A multi-objective optimization formulation of the economic-emission dispatch problem seeks to simultaneously minimize the fuel cost and emission functions subject to the system and network constraints. Note that these constraints are made up of the generation capacity constraint and the power balance constraint. Thus the multi-objective economic-emission dispatch problem is formulated as

$$
\begin{array}{cc}
\text { minimize } & {\left[C\left(\mathbf{P}_{\mathbf{g}}\right), E\left(\mathbf{P}_{\mathbf{g}}\right)\right]} \\
\text { subject to: } & h\left(\mathbf{P}_{\mathbf{g}}\right)=0 \quad \mathbf{P}_{\mathbf{g}}=\left[P_{g_{1}}, \ldots, P_{g_{p}}\right]^{T} \\
& g\left(\mathbf{P}_{\mathbf{g}}\right) \leq 0
\end{array}
$$

where $\mathbf{P}_{\mathbf{g}}$, the decision variable, is the vector of the generated power, $C$ is the fuel cost objective, $E$ is the pollutant emission objective, $g$ and $h$ are the system's equality and the inequality constraints respectively.

\section{Our Approach}

We begin by revisiting the weighted sum approach to solving the multiobjective optimization problem and present an adaptive weighted selection 
scheme. The semi-definite program is then presented along with polynomial optimization relaxation methods.

\subsection{Weighted Sum Method}

Consider the weight vector $\mathbf{w}=\left[w_{1}, \ldots, w_{p}\right]^{T} \in \mathbf{R}^{p}$, the vector objective function $\mathbf{f}(\mathbf{x})=\left[f_{1}(x), \ldots, f_{p}(x)\right]^{T} \in \mathbf{R}^{p}$ and the map $\phi(\mathbf{f}, \mathbf{w}): \mathbf{R}^{p} \times \mathbf{R}^{p} \mapsto \mathbf{R}$. In the weighted sum method a linear or convex combination of the objectives $f_{i}(x), i=1, \ldots, p$ is formed. Each of the objective $f_{i}(x)$ is multiplied by a weight factor $w_{i}$ and then summed up to give the scalar objective, $\phi(\mathbf{f}, \mathbf{w})$, as

$$
\begin{aligned}
\phi(\mathbf{f}, \mathbf{w}) & =\sum_{i=1}^{p} w_{i} f_{i}(x) \\
& =\mathbf{w}^{\mathbf{T}} \mathbf{f}(\mathbf{x})
\end{aligned}
$$

where $p$ is the number of the objectives and

$$
\sum_{i=1}^{p} w_{i}=1, w_{i} \geq 0, i=1, \ldots, p .
$$

This transforms the vector optimization to a scalar of the form:

$$
\begin{aligned}
\operatorname{minimize} & \phi(\mathbf{f}, \mathbf{w}) \\
\text { subject to } & x \in X
\end{aligned}
$$

This process maps the $p$-dimensional objective space onto the positive real line $\mathbf{R}$ and all the optimal (non-dominated) points are mapped to the same point on the line.

As an illustration, consider the bi-objective problem with $p=2$. Equations (16) and (17), respectively, reduce to

$$
\phi(\mathbf{f}, \mathbf{w})=w_{1} f_{1}(x)+w_{2} f_{2}(x)
$$


and

$$
w_{1}+w_{2}=1, w_{1}, w_{2} \geq 0 .
$$

\subsubsection{Adapting Weight Selection in Weighted Sum Method}

If the weights in (19) are parameterized by $\lambda$, such that $w_{1}=\lambda$ and $w_{2}=1-\lambda$, a uniform spacing on $\lambda$ does not produce a uniform spacing on the Pareto front. However, in [38], when the weight is parameterized such that $\lambda$ is constrained on the surface of an ellipsoid, an improvement in distribution of the points on the Pareto front was obtained. In the parameterization, setting

$$
w_{1}=\frac{\lambda_{1}^{2}}{k_{1}^{2}}, w_{2}=\frac{\lambda_{2}^{2}}{k_{2}^{2}}
$$

and substituting (21) in (20), we obtain the equation of the ellipsoid

$$
\frac{\lambda_{1}^{2}}{k_{1}^{2}}+\frac{\lambda_{2}^{2}}{k_{2}^{2}}=1
$$

where $k_{1}$ and $k_{2}$ are the axes of the ellipsoid. The expression can be normalized by setting $k_{2}=1$. This implies that the minor axis of the ellipsoidal surface is set to unit value. However, $k_{1}$ is allowed to take any value greater than 1. Variation in $k_{1}$ allows for the control of the curvature of the ellipsoidal surface. Thus, the non-linear weight selection gives a higher sensitivity and provides for further sensitivity improvement through the free parameter $k_{1}$. This parameter can be manipulated in search of the solution points such that clustered points can be spread out, thereby improving computational efficiency of the method.

\subsection{Semi-definite programming}

The semi-definite program (SDP) is a convex optimization that generalizes the linear program. This is achieved by replacing vector variables with 
matrix variables and the element-wise non-negativity of vectors by positive semi-definiteness of the matrices. There are various SDP forms. In this paper, the primal SDP is defined as the optimization problem

$$
\begin{array}{cl}
\operatorname{minimize} & \left\langle\mathbf{A}_{\mathbf{0}}, \mathbf{X}\right\rangle \\
\text { subject to } & \left\langle\mathbf{A}_{\mathbf{i}}, \mathbf{X}\right\rangle=b_{i}, \quad i=1, \ldots, m \\
& \mathbf{X} \geq 0
\end{array}
$$

and the associated dual SDP is

$$
\begin{aligned}
\operatorname{maximize} & \langle\mathbf{b}, \mathbf{y}\rangle \\
\text { subject to } & \sum_{i=1}^{m} y_{i} \mathbf{A}_{\mathbf{i}} \leq \mathbf{A}_{\mathbf{0}}, \quad \mathbf{y} \in \mathbf{R}^{m}
\end{aligned}
$$

where $\mathbf{X} \in \mathcal{S}^{n}$ is the decision variable, $\mathbf{b} \in \mathbf{R}^{m}$ and $\mathbf{A}_{\mathbf{0}}, \mathbf{A}_{\mathbf{i}} \in \mathcal{S}^{n}$. $\mathcal{S}^{n}$ is the set of all symmetric matrices in $\mathbf{R}^{n \times n}$. The inner product between two vectors $\mathbf{x}, \mathbf{z} \in \mathbf{R}^{n}$ is defined as $\langle\mathbf{x}, \mathbf{z}\rangle=\sum_{i=1}^{n} x_{i} z_{i}$ and the inner product between two matrices $\mathbf{X}, \mathbf{Y} \in \mathcal{S}^{n}$ is defined as $\langle\mathbf{X}, \mathbf{Y}\rangle=\operatorname{Tr}(\mathbf{X} \mathbf{Y})=\sum_{i=1}^{n} \sum_{j=1}^{n} X_{i j} Y_{i j}$. Let $\geq$ denote positive semi-definiteness i.e. $\mathbf{X} \geq \mathbf{Y}$ if $\mathbf{X}-\mathbf{Y} \geq 0$. More details on SDP can be found in [39].

The linear program duality is very strong, so both primal and dual always give the same result. On the other hand, the duality results in SDP are weaker and there exists the duality gap. In this situation, the result of one program yields a bound on the optimal value of the other. However, zero duality gap is achieved if the problem satisfies the Slater's condition [40]. Efficient interior point methods have been developed to solve the primal/dual program $[41,42,43]$.

\subsection{SDP Relaxation of Polynomial Optimization Problem}

We start by introducing some concepts from real algebra that will facilitate our presentation. 
Let $\mathbf{R}[x]$ denote the ring of all real polynomials in the variables $x_{1}, x_{2}, \ldots, x_{n}$ and $\mathcal{P}$ denote the $\mathbf{R}$-vector space spanned by the infinite monomial basis $v \in \mathcal{P}$, given by

$$
v=\left[1, x_{1}, x_{2}, \ldots, x_{n}, x_{1}^{2}, x_{1} x_{2}, \ldots x_{1} x_{n}, x_{2} x_{3}, \ldots\right]^{T} .
$$

We can define a finite monomial basis, $v_{k}$, in $v$ with $\operatorname{deg}\left(v_{k}\right) \leq k$ and this represents a polynomial subspace $\mathcal{P}_{k} \subset \mathcal{P}$. The subset of $\mathbf{R}[x]$ consisting of the sum of squares of polynomials is denoted by $\sum^{2}[x]$. A closed semialgebraic set is denoted by

$$
\mathbf{K}=\left\{x \in \mathbf{R}^{n} \mid g_{i}(x) \geq 0, i=1, \ldots, r\right\}
$$

where $g_{i}(x) \in \mathbf{R}[x], i=1, \ldots, r$. A quadratic module, $M\left(g_{1}, \ldots, g_{m}\right)$, generated by the polynomial $g_{i}(x) \in \mathbf{R}[x], i=1,2, \ldots, m$ is defined as:

$$
M\left(g_{1}, \ldots, g_{m}\right):=\left\{\sigma_{0}+\sum_{j=1}^{m} \sigma_{j} g_{j} \mid \sigma_{j} \in \Sigma^{2}[x]\right\}
$$

The truncated quadratic module of degree $2 k, M_{k}\left(g_{1}, \ldots, g_{m}\right) \subset M\left(g_{1}, \ldots, g_{m}\right)$ have $\operatorname{deg}\left(\sigma_{0}\right) \leq 2 k, \operatorname{deg}\left(\sigma_{i} g_{i}\right) \leq 2 k, i=1, \ldots, m$.

\subsubsection{Polynomial Optimization Problem}

Given a basic closed semi-algebraic set $\mathbf{K}$, the problem of polynomial optimization is that of finding, if possible, or computing a good approximation for the global minimum $p^{*}$ of $p(x) \in \mathbf{R}[x]$

$$
p^{*}=\inf \{p(x) \mid x \in \mathbf{K}\}
$$

and, an optimal value 


$$
x^{*}=\left\{x \mid \forall x \in \mathbf{K}, p\left(x^{*}\right) \leq p(x)\right\}
$$

The problem is generally non-convex with several local minima and hard to solve. Therefore, approximations to the problem are usually considered. Two major forms of convex (semi-definite) relaxations have been proposed in the literature [44]. They are (i) the sum of squares (SOS) method, and (ii) the method of moments.

\subsubsection{Sum of Squares method}

The sum of squares method [45], was an approach developed to reduce the POP problem to finding the largest lower bound, $p^{*}$,

$$
p^{*}=\sup \{\lambda \mid p(x)-\lambda \geq 0, \forall x \in \mathbf{K}\}
$$

The non-negative polynomial $p(x)-\lambda \geq 0$ is then written as sums of squares (or quadratic module) which is transformed into an SDP form as shown below

$$
p^{*}=\sup \left\{\lambda \mid p(x)-\lambda=\sigma_{0}+\sum_{j=1}^{r} \sigma_{j} g_{j} \in \Sigma^{2}[x]\right\}
$$

where $\sigma_{j} \in \Sigma^{2}[x], j=0, \ldots, r$. Various hierarchy of SDP relaxation (approximation) is introduced by setting the polynomial to the truncated quadratic modules $M_{t}\left(g_{1}, \ldots, g_{r}\right)$ such that $\operatorname{deg}\left(\sigma_{0}\right) \leq 2 t, \operatorname{deg}\left(\sigma_{j} g_{j}\right) \leq 2 t$ :

$$
p_{t}^{s o s}=\sup \left\{\lambda \mid p(x)-\lambda=\sigma_{0}+\sum_{j=1}^{r} \sigma_{j} g_{j} \in M_{t}\left(g_{1}, \ldots, g_{r}\right)\right\}
$$

It follows from (30) that $p_{t}^{\text {sos }}$ can be computed through a semi-definite program. And as $t \rightarrow \infty, p_{t}^{s o s} \rightarrow p^{*}$ provided that there exists a number $N \in \mathbf{N}$ such that $N-\|x\|^{2} \in M\left(g_{1}, \ldots, g_{r}\right)$. 


\subsubsection{Method of Moments}

The method of moments is the dual of the SOS method. Its formulation makes use of the theory of moment [46]. This method replaces the problem in (28) with an equivalent convex optimization problem in an infinitedimensional space of measures on $\mathbf{R}^{n}$ :

$$
p^{*}=\min \left\{\int p(x) \mu(d x) \mid \mu \in \mathcal{M}(\mathbf{K})\right\}
$$

The formulation defines a probability measure $\mathcal{M}(\mathbf{K})$ on $\mathbf{K}$, and exchanges every point $x \in \mathbf{K}$ by its Dirac probability measure at $x$. The probability measure $\mu \in \mathcal{M}$ is equipped with the properties $\mu(\emptyset)=0$ and $\mu(\mathbf{K})=1$. As a representing measure, $\mu$ defines the sequence $\mathbf{y}=\left\{\mathbf{y}_{\alpha}\right\}$, called the moment of order $\alpha$, as

$$
y_{\alpha}=\int_{\mathbf{K}} x^{\alpha} d \mu \forall \alpha \in \mathbf{N}^{n}
$$

The sequence $y$ is characterized by its moment matrix, $M(y)$, and the localizing matrix, $M\left(g_{i} y\right)$. Every polynomial $p(x) \in \mathcal{P}$ can be identified by its vector $\mathbf{p}=\left\{p_{\alpha}\right\}_{\alpha \in \mathbf{N}^{n}}$ of coefficient in the infinite basis. To define the above two matrices, define a linear mapping, $L_{y}: \mathcal{P} \mapsto \mathbf{R}$ :

$$
L_{y}(p)=\langle p, y\rangle=\sum_{\alpha \in \mathbf{N}^{n}} p_{\alpha} y_{\alpha}
$$

and a bilinear mapping $\langle\cdot, \cdot\rangle_{y}: p_{\alpha} \times p_{\alpha} \mapsto \mathbf{R}$ :

$$
\begin{aligned}
\langle p, q\rangle_{y} & =L_{y}(p q)=\int\langle p, v\rangle\langle v, q\rangle \mu(d x) \\
& =\int\left\langle p, v v^{T} q\right\rangle d \mu=\left\langle p, \int v v^{T} d \mu q\right\rangle \\
& =\langle p, M(y) q\rangle
\end{aligned}
$$


The moment matrix $M(y)=\int v v^{T} d \mu$ is indexed in the infinite basis $v$. Let $v_{k}$ denote the finite basis of the subspaces $\mathcal{P}_{k} \subset \mathcal{P}$ of real polynomial with $\operatorname{deg}\left(\mathcal{P}_{k}\right) \leq k$. Then, for all $p(x), q(x) \in \mathcal{P}_{k}, M_{k}(y)=\int v_{k} v_{k}^{T} d \mu$. It follows that if $y$ has a representing measure, then $M_{k}(y) \geq 0, k=0,1, \ldots$. Consider $g(x) \in \mathcal{P}, g(x)=\sum_{\alpha} g_{\alpha} x^{\alpha}$. The bilinear mapping associated with $g y$ is

$$
\langle p, q\rangle_{g y}=L_{y}(g p q)=\langle p, M(g y) q\rangle,
$$

where $M(g y)$ is called the localizing matrix associated with $y$ and $g$. For all polynomials in $\mathcal{P}_{k}, M_{k}(g y) \geq 0$ for all $k$.

A finite-dimensional relaxation of the problem can now be defined. For $\max \left(\operatorname{deg} p(x), \max g_{i}\right) \leq 2 k$, a semi-definite program equivalent of (28) is

$$
\begin{array}{ll}
p_{k}^{\text {mom }}= & \inf y^{T} p \\
\text { s.t. } & y_{0}=1, M_{k}(y) \geq 0 \\
& M_{k-d_{i}}\left(g_{i} y\right) \geq 0, i=1, \ldots, r
\end{array}
$$

where $d_{i}=\operatorname{deg}\left(g_{i}\right)$. The problem in (40) can be clearly seen as an SDP relaxation of order $k$ of the problem in (33) by writing $M_{k}(y)=\sum_{\alpha} B_{\alpha} y_{\alpha}$ and $M_{k-d_{i}}\left(g_{i} y\right)=\sum_{\alpha} C_{\alpha}^{i} y_{\alpha}, i=1, \ldots, r$ with appropriate symmetric matrices $B_{\alpha}, C_{\alpha}^{i}$. The SDP dual of (40) is the LMI problem

$$
\begin{array}{ll} 
& \max _{\lambda, X, Z_{i}} \lambda \\
\text { s.t. } & \left\langle B_{0}, X\right\rangle+\sum_{i=1}^{r}\left\langle C_{0}^{i}, Z_{i}\right\rangle=p(x)-\lambda \\
& \left\langle B_{\alpha}, X\right\rangle+\sum_{i=1}^{r}\left\langle C_{\alpha}^{i}, Z_{i}\right\rangle=p_{\alpha}, i=1, \ldots, r ;|\alpha| \leq 2 k \\
& X \geq 0, Z_{i} \geq 0, i=1, \ldots, r
\end{array}
$$

and with $X, Z_{i} \in \Sigma^{2}[x]$, problem (41) can be written as problem (32). The two programs (30) and (33) give the dual formulation for the polynomial 
(28), while the programs (40) and (41) are the SDP dual. By weak duality $p_{k}^{\text {sos }} \leq p_{k}^{\text {mom }} \leq p^{*}$, and equality $p_{k}^{\text {sos }}=p_{k}^{\text {mom }}$ when the set $\mathbf{K}$ is strictly feasible. Although the two approaches are dual of one another, it has been observed that not every non-negative polynomial has sum of squares equivalent.

In general, the SDP relaxations with both methods generate a large number of variables, and their computations involve large matrix dimension. This limits the application of the SDP approach to small and medium sized problems. Extending the application of the SOS formulation has been the interest of recent research work. In [47, 48], a method that exploits the sparsity structure of the problem to reduce the dimension of the problem was proposed. Another method that exploits the symmetry of the problem matrices was considered in [49]. Generally, it is not always possible to achieve exact SDP relaxation of the original problem, especially for large and non convex problem. This implies that the SDP solution only provides a bound for the optimal solution. There has been efforts to ensure zero duality with non convex problems like the optimal flow problems (OPF) [29].

\section{EED Problem Formulated as POP}

The emission function model is highly non-linear and it is generally expressed as a combination of polynomial and exponential parts $[5,2]$. Furthermore, the class of polynomial functions is the only class for which algorithms that guarantee infimum has been developed [50]. Hence, it is reasonable to express the exponential part in power series form. We note that the exponential function is contained in an infinite dimensional polynomial space; but using the result in [51], the problem can be projected into a finite dimensional 
space. This projection reduces the infinite dimensional polynomial problem to the standard polynomial optimization problem (POP) form which can be efficiently solved via semi-definite programming (SDP) [46].

Returning to the EED problem as stated in (15), the objective function $C\left(\mathbf{P}_{\mathbf{g}}\right)$ is a finite degree polynomial but $E\left(\mathbf{P}_{\mathbf{g}}\right)$, due to the exponential part, is a polynomial with an infinite monomial basis. If $P_{g_{i}}=x_{i}, C\left(\mathbf{P}_{\mathbf{g}}\right)$ can be written as $C(\mathbf{x})$ and $E\left(\mathbf{P}_{\mathbf{g}}\right)$ as $E(\mathbf{x})$.

\subsection{Polynomial Approximation of $e^{x}$}

The exponential function can be expressed using the Maclaurin series expansion:

$$
e^{x}=\sum_{i=1}^{\infty} \frac{x^{i}}{i !} .
$$

This expression shows that the exponential function resides in an infinite di-

mensional space spanned by the infinite monomial basis $\left\{1, x, x^{2}, \ldots, x^{k}, \ldots\right\}$. Such space is rather complex to handle numerically and so the exponential function should be projected unto finite dimensional space in order to obtain reasonable solution that is close to the exact value. By fixing the degree $k$, the optimal polynomial which gives the best approximating error for the exponential function in the emission function can be computed using the Gram-Schmidt procedure [52] or equivalently by the least square error approximation. The substitution of this optimum value in equation (7), reduces $E(\mathbf{x})$ to a polynomial of degree $k$.

Using the results in [51] and fixing the degree $k$, an optimal representation, $E_{k}^{o}(\mathbf{x})$, is computed for $E(\mathbf{x})$ using the MATLAB polynomial least square error function polyfit(). The optimal polynomial approximation of 
degree 2, 4 and 6 were determined for the exponential functions. The set of polynomial approximations of the exponential function, for generator $P_{3}$, with even degrees are as shown in Fig. 1. It can be observed that the polynomial functions fit the exponential function more closely as the degree of the polynomials increases. Also, considering the size of the norm of the residual of the quadratic approximation, it can be concluded that the quadratic approximation is a poor approximation of the exponential function of $P_{3}$. The relative errors (or residuals) of the approximation polynomials of degree 2, 4 and 6 are as presented Table 1. Each weighted exponential function, $\zeta_{i} \exp \left(\lambda_{i} x_{i}\right)$, is represented as

$$
\zeta_{i} \exp \left(\lambda_{i} x_{i}\right) \approx \sum_{j=0}^{k} a_{i_{j}} x_{i}^{j}
$$

Equation (7) thus becomes

$$
E_{k}^{o}(\mathbf{x}) \approx \sum_{i=1}^{p}\left(\left(\alpha_{i}+\beta_{i} x_{i}+\gamma_{i} x_{i}^{2}\right)+\sum_{j=0}^{k} a_{i_{j}} x_{i}^{j}\right),
$$

Also, all the constraints in (10)-(12) are quadratic or linear in the decision variable $x$. Thus the EED problem reduces to a multi-objective polynomial optimization problem (MOPOP) that can be written as

$$
\begin{array}{cc}
\text { minimize } & {\left[C(\mathbf{x}), E_{k}^{o}(\mathbf{x})\right]} \\
\text { subject to: } & h(\mathbf{x})=0 \quad \mathbf{x}=\left[x_{1}, \ldots, x_{p}\right]^{T} \\
& g(\mathbf{x}) \leq 0 .
\end{array}
$$

The formulation in (45) captures the general EED problems whose objective functions are expressed, or can be approximated, as polynomial functions.

The MOPOP is further reduced to a scalar (or standard) POP by the application of the weighted sum method which linearly combines the two 
objectives, $\phi(\mathbf{x}, \mathbf{w})=w_{1} C(\mathbf{x})+w_{2} E_{k}^{o}(\mathbf{x})$. By converting the equality constraint $h(\mathbf{x})=0$ into two inequality constraints, $h(\mathbf{x}) \geq 0$ and $h(\mathbf{x}) \leq 0$, the problem thus takes the standard POP form:

$$
\begin{array}{cl}
\text { minimize } & \phi(\mathbf{x}, \mathbf{w}) \\
\text { subject to: } & h(\mathbf{x}) \leq 0 ; \quad h(\mathbf{x}) \geq 0 \\
& g(\mathbf{x}) \leq 0
\end{array}
$$

To solve the resulting POP, we rely on SparsePOP, an efficient POP algorithm code.

SparsePOP is a freely available MATLAB software that implements POP solution algorithm based on the SOS method [53]. It exploits a sparse structure of polynomials in POPs while applying a hierarchy of SDP relaxations of increasing dimension whose associated monotone sequence of optimal values converge to the global value. Unlike the moment method whose relaxation on the degree of the polynomials, its relaxations depends on the supports of the polynomials [47]. It solves the resulting SDP problem using SeDuMi as the default solver [54].

\section{Simulation Results and Comparative Analysis}

The proposed algorithm was evaluated on some standard test systems to investigate the effectiveness of the approach. Two cases of this are considered, namely:

1. the general EED with quadratic fuel cost and emission function with exponential part,

2. the problem with both the fuel and the emission functions expressed as cubic polynomial functions. 


\subsection{Case 1}

For this case, the test system is the standard IEEE 30-bus 6-unit system with the total real load of 283.4 MW. The cost coefficients, emission coefficients and power generation limits are given in Table 2. Details of the bus and line data can be found in [55]. To be able to compare the generated result with those reported in the literature, the problem was solved with the transmission loss neglected. Two different values of polynomial degree of approximations $(k=4,6)$ for the exponential function were considered. The problem was initially solved for the ideal minimum points with each of the single objectives. The fuel cost and the emission functions were, using the weighted sum method, combined to give a single scalar objective, thus reducing the problem to a single objective POP. SparsePOP was applied to the resulting 4-degree and 6-degree POP. In order to explore the Pareto front, twenty-one runs were conducted on each of the polynomial approximates after normalization. The results for the best fuel cost and the best emission objectives against those reported using LP [21], strength Pareto Evolutionary Algorithm (SPEA) [15], non-dominated sorting genetic algorithm-II (NSGAII) [56] and niched Pareto genetic algorithm (NPGA) [9] are shown in Tables 3 and 4. The combined generated Pareto fronts for the 4- and 6-degree polynomial approximations is shown in Fig. 2. No obvious differences can be observed in the Pareto fronts generated for the two polynomial approximations considered. However, the solutions using polynomial of degree 6 can be observed to generate solutions that slightly dominates those of degree 4 looking at the Tables 3 and 4 . When compared with those reported in the literature, its solutions are not dominated. This confirms its good convergence 
characteristic and the global optimality of its solutions.

The problem is also solved with the inclusion of transmission losses in the power balance constraints. Twenty-one runs were conducted for the 6th degree polynomials. The generated non-dominated Pareto set along with the no-loss case is presented in Fig. 3. Tables 5 and 6 show the comparison of the solutions for minimum fuel cost and minimum emission, respectively, with those reported in the literature. In Fig. 3, a shift of the Pareto curve in the direction of the cost axis can be observed relative to the result obtained in the no-loss case. This can be attributed to the cost of the power lost through the transmission network.

In Figures 2 and 3, a clustering of the solution points on the Pareto front with even change in the weight factor can be observed. A non-linear weight adaptation proposed in (21) is applied on the test problem using the 6th degree approximation polynomial with transmission losses included in the power balance constraint. The effect of the factor $k_{1}$ on the diversity of solution generated is shown in Fig. 4. It is quite apparent that the proposed weight adaptation improves the uniform distribution of the solutions as the value of $k_{1}$ increases. On the other hand, as the value of $k_{1}$ increases, a reduction in the number of points captured by the SDP approach, around the lower extreme point, can also be observed. This leads to a reduction of the extent of the generated solutions. An optimal value of $k_{1}$ that gives a good distribution and comparatively good extent can be empirically determined.

\subsection{Case 2}

In this case study, the method is extended to solve EED problem with the fuel cost and the emission objective functions expressed as cubic functions. 
The total fuel cost is given as

$$
C\left(\mathbf{P}_{\mathbf{g}}\right)=\sum_{i=1}^{p} a_{i}+b_{i} P_{g_{i}}+c_{i} P_{g_{i}}^{2}+d_{i} P_{g_{i}}^{3},
$$

where $C$ is the total fuel cost, $P_{g_{i}}$ is the real power output of the $i^{\text {th }}$ plant, and $a_{i}, b_{i}, c_{i}$ and $d_{i}$ are the fuel cost coefficients of that plant. The emission function is given as

$$
E\left(P_{g}\right)=\sum_{i=1}^{p}\left(\alpha_{i}+\beta_{i} P_{g_{i}}+\gamma_{i} P_{g_{i}}^{2}+\delta_{i} P_{g_{i}}^{3}\right),
$$

where $\alpha_{i}, \beta_{i}, \gamma_{i}, \lambda_{i}$ and $\delta_{i}$ are the coefficients of the $i^{\text {th }}$ plant emission characteristics. Under this consideration, two test systems are considered: a 6-unit system and a 13-unit system. For the purpose of comparison with the methods presented in [31] and [32], the objectives were aggregated into a single cost objective by monetizing the emission generated. This is achieved through the use of price penalty factor, $q_{i}$, which gives money equivalent of the generated emission. The price penalty factor is given as the ratio of the maximum fuel cost of a thermal unit and its maximum emission. This is expressed as:

$$
q_{i}=\frac{C_{i}\left(P_{i_{\max }}\right)}{E_{i}\left(P_{i_{\max }}\right)}
$$

This objective function then reduces to

$$
\phi\left(P_{g i}, w\right)=\sum_{i=1}^{p} w_{1} C_{i}\left(P_{g i}\right)+w_{2} q_{i} E_{i}\left(P_{g i}\right)
$$

\subsubsection{6-Unit Test System}

The test system is a 30-bus 6-unit system with cubic fuel and emission functions. The fuel cost coefficients, emission coefficients and the real power 
limit of the generators are as given in [31]. The problem was scalarized and solved for different load demand values between 150 - 250MW.

In [31], different price penalty factors were investigated on the test system, and it was noted that min-max price penalty factor gave the best performance. Also, the multi-objective problem was solved with the assumption that the individual objectives are equally weighted. This implies a weight factor of $w_{1}\left(=w_{2}\right)=0.5$. Therefore, solutions provided by the SDP method are compared with those given by the min-max price penalty factor.

In solving the problem using the SDP method, the unit's emission function for $\mathrm{CO}_{2}-E_{i C O_{2}}, \mathrm{NO}_{x}-E_{i N O_{x}}$ and $\mathrm{SO}_{2}-E_{i \mathrm{SO}_{2}}$, were combined into one emission function, $E_{i}$. The problem was solved for the minimum fuel cost and minimum emission. Table 7 shows SDP solutions for minimum fuel cost and minimum emission, respectively, with the best values presented in bold. Also for comparison with the results presented in [31], the problem was solved for a weight factor of $w_{1}=0.5$ for the different values of $P_{D}$ considered. The comparison of the SDP solutions with those presented in [31] using the Langrangian (LAN) method is as shown Table 8. The superiority of the SDP method can be seen in the lower fuel cost and emission in Table 8 for all values of load considered.

\subsubsection{3-Unit Test System}

The proposed method was also tested on a 13-unit test system considered in [32]. Details of the system can be found therein. The problem specified the spinning reserve requirements. The problem was solved for a load demand of $1900 \mathrm{MW}$ with 11 different weight factors taking into account the line losses. The solutions of the SDP method in comparison with those of the 
evolutionary methods (GA, PSO, BF and an hybridized BF with NelderMead BF-NM) presented in [32] are as shown in Table 9. It can be observed from Table 9 that the solution produced by the SDP method dominates those of the heuristic methods for all values of the weighting factors.

\section{Conclusion}

The multi-objective economic-emission dispatch problem with transmission losses is formulated as a convex optimization problem through SDP relaxation technique. A convex (linear) combination of the polynomial objectives reduced the multi-objective problem to a scalar variation. A free MATLAB software, SparsePOP, that efficiently solves POP was employed. Although the weighted sum method is known to have problem handling nonconvex Pareto front, SDP-based weighted sum shows good convergence property and better exploration of the Pareto front was achieved through function objective normalization.

Numerical examples are considered which show that the proposed formulation is efficient. It shows superior performance over some of the well-known evolutionary algorithms and was observed to have comparable diversity and better convergence properties.

\section{References}

[1] A. A. El-keib, H. Ma, J. L. Hart, Economic dispatch in view of the clean air act of 1990, IEEE TRANS. POWER SYST. 9 (2) (1994) 972-978.

[2] J. H. Talaq, F. El-Hawary, M. E. El-Hawary, A summary of environ- 
mental/economic dispatch algorithms, IEEE TRANS. POWER SYST. 9 (3) (1994) 1508-1516.

[3] J. Resek, K. D. Le, C. J. S. J. L. Golden, R. L. Vice, J. T. Wood, J. ballance, G. Brown, J. Y. Kamya, E. K. Nielsen, H. Nakajima, M. Ookubo, I. Iypda, G. W. Cauley, Potential impacts of clean air regulations on system operations, IEEE Transactions on Power Systems 10 (2) (1995) $647-656$.

[4] J. W. Lamont, E. V. Obessis, Emission dispatch models and algorithms for the 1990's, IEEE TRANS. POWER SYST. 10 (2) (1995) 941-947.

[5] M. R. Gent, J. W. Lamont, Minimum-emission dispatch, IEEE TRANS. POWER APP. SYS. PAS-90 (1971) 2650-2660.

[6] R. L. Sullivan, D. F. Hackett, Air quality control using a minimum pollution-dispatching algorithm, Environmental Science \& Technology 7 (11) (1973) 1019-1022.

[7] J. K. Delson, Controlled emission dispatch, IEEE Transactions on Power Systems 93 (1974) 1359-1366.

[8] J. Zahavi, L. Eisenberg, Economic-environmental power dispatch, IEEE Transactions on Systems, Man \& Cybernetics SMC-5 (1975) 485-489.

[9] M. A. Abido, A niched pareto genetic algorithm for multiobjective environmental/ economic dispatch, Int. Jour. Elect. Power Ener. Sys. 25 (2) (2003) 97-105. 
[10] K. Deb, Multi Objective Optimization using Evolutionary Algorithm, John Wiley and Sons, Ltd, New York, 2004.

[11] J. S. Dhillon, S. C. Parti, D. P. Kothari, Stochastic economic emission load dispatch, Elec. Power Sys. Res. 26 (1993) 179-186.

[12] K. Miettinen, Nonlinear multiobjective Optimization, 1st Edition, Kluwer Academic Publishers, 1999.

[13] R. Yokoyama, S. Bae, T. Morita, H. Sasaki, Multiobjective generation dispatch based on probability security criteria, IEEE Trans. Power Syst. 3 (1) (1988) 317-324.

[14] M. A. Abido, A novel multiobjective evolutionary algorithm for environmental/economic power dispatch, Elec. Power Sys. Res. 65 (1) (2003) 71-81.

[15] M. A. Abido, Environmental/economic power dispatch using multiobjective evolutionary algorithms, IEEE Trans. Power Syst. 18 (4) (2003) 1529-1537.

[16] J. B. Park, K. S. Lee, J. R. Shin, K. Y. Lee, A particle swarm optimization for economic dispatch with nonsmooth cost function, IEEE Trans. Power Syst. 20 (1) (2005) 34-42.

[17] J. G. Vlachogiannis, K. Y. Lee, Economic load dispatch a comparative study on heuristic optimization techniques with an improved coordinated aggregationbased pso, IEEE Trans. Power Syst. 24 (2) (2009) 9911001. 
[18] I. A. Farhat, M. E. El-Hawary, Multi-objective economic-emission optimal load dispatch using bacterial foraging algorithm, 25th IEEE Canadian Conf. Elect. and Comp. Eng. (CCECE), 2012, pp. 1-5.

[19] R. allah Hooshmand, M. Parastegari, M. J. Morshed, Emission, reserve and economic load dispatch problem with non-smooth and non-convex cost functions using the hybrid bacterial foraging-nelder mead algorithm, Appl. Ener. 89 (1) (2012) 443-453.

[20] K. K. Mandal, N. Chakraborty, Effect of control parameters on differential evolution based combined economic emission dispatch with valvepoint loading and transmission loss, Int J. Emerg. Elect Power Sys. 9 (4) (2008) 1-18.

[21] A. Farag, S. Al-Baiyat, T. C. Cheng, Economic load dispatch multiobjective optimization procedures using linear progamming techniques, IEEE TRANS. POWER SYST. 10 (2) (1995) 731-738.

[22] P. A. Parrilo, Semidefinite programming relaxation for semialgebraic problems, Math. Prog., Ser. B 96 (2003) 293-320.

[23] S. Boyd, L. Vandenberghe, Semidefinite programming relaxations of non-convex problems in control and combinatorial optimization, in: A. Paulraj, V. Roychowdhuri, C. Schaper (Eds.), Communications, Computation, Control and Signal Processing: A Tribute to Thomas Kailath, Kluwer, 1997, Ch. 15, pp. 279-288.

[24] J.-B. Lasserre, T. Prieto-Rumeau, Sdp vs. lp relaxations for the mo- 
ment approach in some performance evaluation problems, Stoch. Models 20 (4) (2004) 439-456.

[25] R. Fuentes-Loyola, V. H. Quintana, Medium-term hydothermal coordination by semidefinite programming, IEEE TRANS. POWER SYST. 18 (4) (2003) 1515-1522.

[26] M. Madrigal, H. Quintana, Semidefinite programming relaxations for 0,1 power dispatch problems, in: Proc. IEEE Power Eng. Soc. Summer Meeting Conf., Edmonton, Canada, 1999, pp. 697-701.

[27] B. C. Lesieutre, D. K. Molzahn, A. R. Borden, C. L. DeMarco, Examining the limits of the application of semidefinite programming to power flow problems, in: Forty-Ninth Annual Allerton Conference, Allerton House, UIUC Illinois, USA, 2011, pp. 1492-1499.

[28] R. A. Jabr, Solution to economic dispatching with disjoint feasible regions via semidefinite programming, IEEE TRANS. POWER SYST. 27 (1) (2012) 572-573.

[29] J. Lavaei, S. H. Low, Zero duality gap in optimal power flow problem, IEEE TRANS. POWER SYST. 27 (1) (2012) 92-107.

[30] X. Bai, H. Wei, K. Fujisawa, Y. Wang, Semidefinite programming for optimal power flow problem, Int. Jour. Elect. Power Ener. Sys. 30 (2008) 383-392.

[31] S. Krishnamurthy, R. Tzoneva, Impact of price penalty factors on the solution of the combined economic emission dispatch problem using cubic criterion functions, 2012. 
[32] R.-A. Hooshmand, M. Parastegari, M. J. Morshed, Emission, reserve and economic load dispatch problem with non-smooth and non-convex cost functions using the hybrid bacterial foraging-neldermead algorithm, Appl. Ener. 89 (2012) 443-453.

[33] G. Chesi, Lmi techniques for optimization over polynomials in control: A survey, IEEE TRANS. AUTOMAT. CONTR. 55 (11) (2010) 25002510 .

[34] J. B. Lassarre, A semidefinite programming approach to the generalized problem of moments, Math. Prog. 112 (Ser. B) (2008) 65-92.

[35] S. Hemamalini, S. P. Simon, Maclaurin series-based lagrangian method for economic dispatch with valve-point effect, IET Generation, Transmission and Distribution 3 (9) (2009) 859871.

[36] R. G. Kavasseri, P. Nag, A computational algebraic geometry based global optimization technique to address economic dispatch, in: Proc. IEEE Engineering Society, General Meeting, Tampa,FL, 2007.

[37] G. S. Piperagkas, A. G. Anastasiadis, N. D. Hatziargyriou, Stochastic pso-based heat and power dispatch under environmental constraints incorporating chp and wind power units, Electric Power Systems Research.

[38] A. M. Jubril, A nonlinear weight selection in weighted sum for convex multiobjective optimization, FACTA UNIV.(NIS) Series Maths. and Inform. 27 (3) (2013) 357-372.

[39] L. Vandenberghe, S. Boyd, Semidefinite programming, SIAM Review 38 (1996) 49-95. 
[40] L. Tunçel, On the Slater condition for the sdp relaxations of nonconvex sets, Tech. Rep. CORR 2000-13, University of Waterloo (February 2000).

[41] I. A. Farhat, M. E. El-Hawary, Interior point methods application in optimum operational scheduling of electric power systems, IET Gen., Trans. and Distr. 3 (11) (2009) 1020-1029.

[42] F. Alizadeh, Interior point methods in semidefinite programming with application to combinatorial optimization, SIAM Jour. Opt. 5 (1995) $13-51$.

[43] A. S. Nemirovski, M. J. Todd, Interior-point methods for optimization, Acta Numerica (2008) 191-234.

[44] M. Laurent, Sums of Squares, Moment Matrices and Polynomial Optimization, Vol. 149 of Emerg. Appl. Alge. Geom., IMA, 2009, Ch. 3, pp. $157-270$.

[45] P. A. Parrilo, Structured semidefinite programs and semialgebraicgeometry methods in robustness and optimization, Ph.D. thesis, California Institute of Technology (May 2000).

[46] J. B. Lasserre, Global optimization with polynomials and the problem of moments, SIAM Jour. Opt. 11 (3) (2001) 796-817.

[47] S. Kim, M. Kojima, H. Waki, Generalized lagrangian duals and sums of squares relaxations of sparse polynomial optimization problems, SIAM Jour. Opt. 15 (3) (2005) 697719. 
[48] H. Waki, S. Kim, M. Kojima, M. Muramatsu, Sums of squares and semidefinite programming relaxations for polynomial optimization problems with structured sparsity, SIAM Jour. Opt. 17 (1) (2006) 218-242.

[49] K. Gaterman, P. Parrilo, Symmetry groups, semidefinite programs and sums of squares, Jour. Pure and Appl. Alg. 192 (2004) 95-128.

[50] D. Jibetean, Global optimization of rational multivariate functions, Tech. Rep. PNA-R0120, Centrum voor Wiskunde en Informatica, PNA (October 2001).

[51] O. Devolder, F. Glineur, Y. Nesterov, Solving infinite-dimensional optimization problems by polynomial approximation, discussion paper 2010/29, Universite catholique de Louvain, Center for Operations Research and Econometrics, Belgium (June 2010).

[52] D. G. Luenberger, Optimization by Vector Space Methods, 1st Edition, Willey-Interscience, John Willey and Sons, Inc., 1968.

[53] H. W. S. Kim, M. Kojima, M. M. H. Sugimoto, M. Yamashita, User Manual for SparsePOP: a Sparse Semidefinite Programming Relaxation of Polynomial Optimization Problems (2009).

[54] J. F. Sturm, the Advanced Optimization Laboratory at McMaster University Canada, SeDuMi version 1.1R3, Advanced Optimization Laboratory at McMaster University, Canada (October 2006).

[55] M. A. Abido, Multiobjective evolutionary algorithms for electric power dispatch problem, IEEE Trans. Evol. Comput. 10 (3) (2006) 315-329. 
[56] R. T. F. A. King, H. C. S. Rughooputh, K. Deb, Evolutionary multiobjective environmental/ economic dispatch: Stochastic vs deterministic approaches, Tech. Rep. 2004019, KanGAL (2004). 


\section{List of Figures}

1 Approximating polynomials of degrees 2,4 and $6 \ldots 37$

2 Pareto front for degree 6 polynomial approximation without Transmission Losses . . . . . . . . . . . . . . . . . . . . . 39

3 Pareto front for degree 6 polynomial approximation with and without Transmission Losses . . . . . . . . . . . . . . . . 39

4 Effect of variation of $k_{1}$ on the distribution of the Pareto front 46 


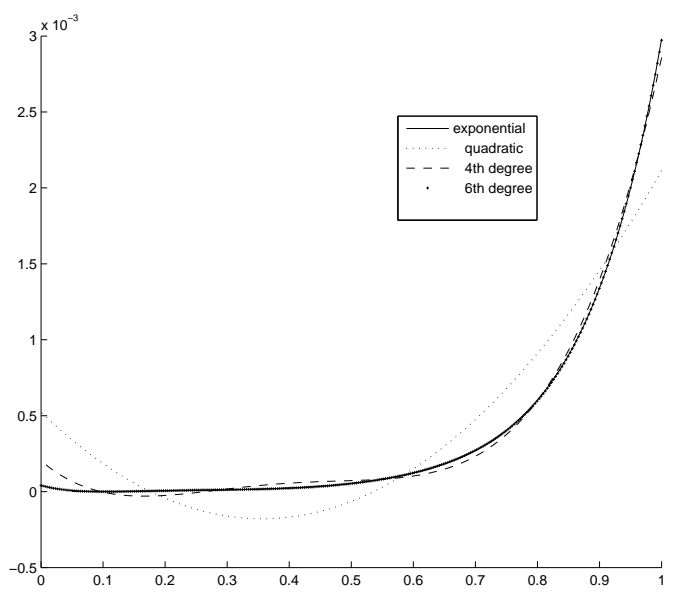

Figure 1: Approximating polynomials of degrees 2, 4 and 6 


\section{List of Tables}

1 Relative residuals of the approximations of different degrees . 40

2 Generator Coefficients and Real Power Limits . . . . . . . . . 41

3 Best Solutions for Fuel Cost without Transmission Losses . . . 42

4 Best Solutions for Emission without Transmission Losses . . . 43

$5 \quad$ Best Solutions for Fuel Cost Case with Transmission Losses . . 44

6 Best Solutions for Emission with Transmission Losses . . . . . 45

7 Summary of best solutions for the 6 -unit test system . . . . . 46

8 Solution of the 6-unit test bus with equally weighted objectives 47

9 The values of the aggregated objective function obtained by different methods for different values of the weight factors . . 47 


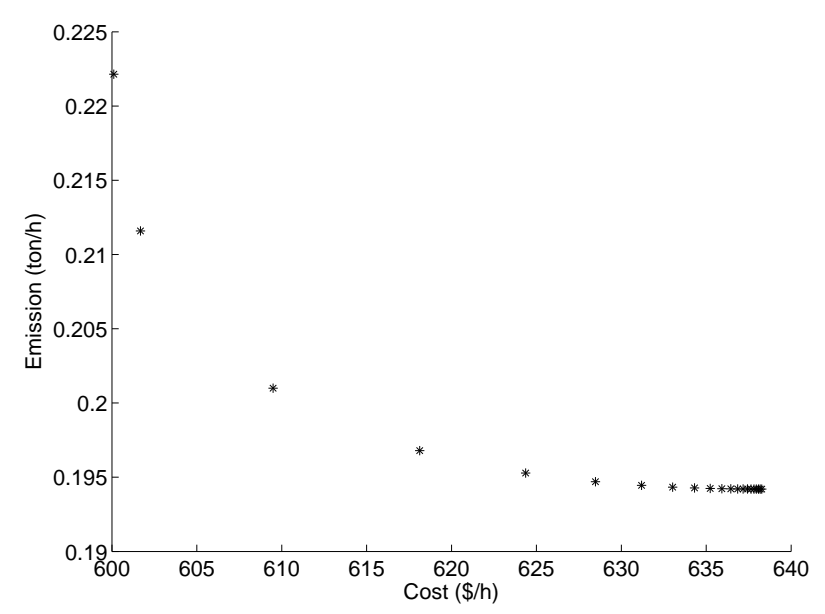

Figure 2: Pareto front for degree 6 polynomial approximation without Transmission Losses

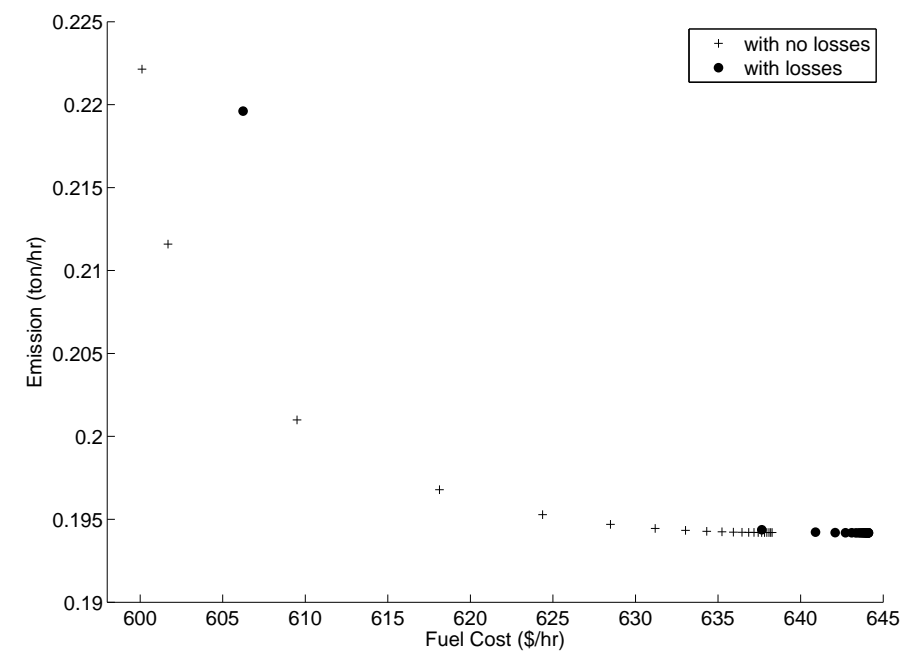

Figure 3: Pareto front for degree 6 polynomial approximation with and without Transmission Losses 
Table 1: Relative residuals of the approximations of different degrees

\begin{tabular}{ccccccc}
\hline degree & P1 & P2 & P3 & P4 & P5 & P6 \\
\hline 2 & $3.12 \cdot 10^{-5}$ & $3.13 \cdot 10^{-4}$ & $2.25 \cdot 10^{-3}$ & $3.01 \cdot 10^{-3}$ & $2.25 \cdot 10^{-3}$ & $1.74 \cdot 10^{-4}$ \\
4 & $1.65 \cdot 10^{-7}$ & $3.33 \cdot 10^{-6}$ & $3.20 \cdot 10^{-5}$ & $4.99 \cdot 10^{-5}$ & $3.21 \cdot 10^{-4}$ & $7.01 \cdot 10^{-6}$ \\
6 & $6.91 \cdot 10^{-10}$ & $1.69 \cdot 10^{-8}$ & $2.45 \cdot 10^{-5}$ & $3.96 \cdot 10^{-7}$ & $2.45 \cdot 10^{-5}$ & $1.38 \cdot 10^{-7}$ \\
\hline
\end{tabular}


Table 2: Generator Coefficients and Real Power Limits

\begin{tabular}{cllllll}
\hline & $P_{1}$ & $P_{2}$ & $P_{3}$ & $P_{4}$ & $P_{5}$ & $P_{6}$ \\
\hline$a$ & 10 & 10 & 20 & 10 & 20 & 10 \\
\hline \multicolumn{1}{c}{200} & 150 & 180 & 100 & 180 & 150 \\
\hline$\alpha$ & 100 & 120 & 40 & 60 & 40 & 100 \\
\hline$\beta$ & -5.554 & -6.047 & -5.094 & -3550 & -5.094 & -5.555 \\
\hline$\gamma$ & 6.490 & 5.638 & 4.586 & 3.380 & 4.586 & 5.151 \\
\hline$\zeta$ & $2.0 \cdot 10^{-4}$ & $5.0 \cdot 10^{-4}$ & $1.0 \cdot 10^{-6}$ & $2.0 \cdot 10^{-3}$ & $1.0 \cdot 10^{-6}$ & $1.0 \cdot 10^{-5}$ \\
\hline$\lambda$ & 2.857 & 3.333 & 8.000 & 2.000 & 8.000 & 6.667 \\
\hline$P_{g}^{\text {min }}$ & 0.05 & 0.05 & 0.05 & 0.05 & 0.05 & 0.05 \\
\hline$P_{g}^{\text {max }}$ & 0.50 & 0.60 & 1.00 & 1.20 & 1.00 & 0.60 \\
\hline
\end{tabular}


Table 3: Best Solutions for Fuel Cost without Transmission Losses

\begin{tabular}{ccccccc} 
& SDP & SDP & LP & NPGA & SPEA & NSGA-II \\
\hline & 4 th & 6 th & & & & \\
\hline$P_{g_{1}}$ & 0.1097 & 0.1097 & 0.1500 & 0.1080 & 0.1062 & 0.1050 \\
$P_{g_{2}}$ & 0.2998 & 0.2998 & 0.3000 & 0.3284 & 0.2897 & 0.3177 \\
$P_{g_{3}}$ & 0.5243 & 0.5243 & 0.5500 & 0.5386 & 0.5289 & 0.5216 \\
$P_{g_{4}}$ & 1.0162 & 1.0162 & 1.0500 & 1.0067 & 1.0025 & 1.0146 \\
$P_{g_{5}}$ & 0.5243 & 0.5243 & 0.4600 & 0.4949 & 0.5402 & 0.5159 \\
$P_{g_{6}}$ & 0.3597 & 0.3597 & 0.3500 & 0.3574 & 0.3664 & 0.3583 \\
\hline Cost & $\mathbf{6 0 0 . 1 1 1 4}$ & $\mathbf{6 0 0 . 1 1 1 4}$ & $\mathbf{6 0 4 . 1 5}$ & $\mathbf{6 0 0 . 2 5 9}$ & $\mathbf{6 0 0 . 1 5}$ & $\mathbf{6 0 0 . 1 5 5}$ \\
Emi & 0.2222 & 0.22214 & 0.2233 & 0.22116 & 0.2215 & 0.22188 \\
\hline
\end{tabular}


Table 4: Best Solutions for Emission without Transmission Losses

\begin{tabular}{ccccccc} 
& SDP & SDP & LP & NPGA & SPEA & NSGA-II \\
\hline & 4 th & 6 th & & & & \\
\hline$P_{g_{1}}$ & 0.4051 & 0.4062 & 0.4000 & 0.4002 & 0.4116 & 0.4077 \\
$P_{g_{2}}$ & 0.4581 & 0.4592 & 0.4500 & 0.4474 & 0.4532 & 0.4577 \\
$P_{g_{3}}$ & 0.5406 & 0.5377 & 0.5500 & 0.5166 & 0.5329 & 0.5389 \\
$P_{g_{4}}$ & 0.3809 & 0.3832 & 0.4000 & 0.3688 & 0.3832 & 0.3837 \\
$P_{g_{5}}$ & 0.5406 & 0.5377 & 0.5500 & 0.5751 & 0.5383 & 0.5352 \\
$P_{g_{6}}$ & 0.5087 & 0.5101 & 0.5000 & 0.5259 & 0.5148 & 0.5110 \\
\hline Cost & 638.300 & 638.269 & 639.600 & 639.182 & 638.51 & 638.269 \\
Emission & $\mathbf{0 . 1 9 4 2}$ & $\mathbf{0 . 1 9 4 2}$ & $\mathbf{0 . 1 9 4 2}$ & $\mathbf{0 . 1 9 4 3}$ & $\mathbf{0 . 1 9 4 2}$ & $\mathbf{0 . 1 9 4 2 0}$ \\
\hline
\end{tabular}


Table 5: Best Solutions for Fuel Cost Case with Transmission Losses

\begin{tabular}{ccccc} 
& SDP & NPGA & SPEA & NSGA-II \\
\hline$P_{g_{1}}$ & 0.1134 & 0.1245 & 0.1086 & 0.1182 \\
$P_{g_{2}}$ & 0.2990 & 0.2797 & 0.3056 & 0.3142 \\
$P_{g_{3}}$ & 0.5977 & 0.6284 & 0.5818 & 0.5910 \\
$P_{g_{4}}$ & 0.9737 & 1.0264 & 0.9846 & 0.9710 \\
$P_{g_{5}}$ & 0.5218 & 0.4693 & 0.5288 & 0.5172 \\
$P_{g_{6}}$ & 0.3546 & 0.3993 & 0.3584 & 0.3548 \\
\hline Cost & $\mathbf{6 0 6 . 2 3 4 8}$ & $\mathbf{6 0 8 . 1 4 7}$ & $\mathbf{6 0 7 . 8 0 7}$ & $\mathbf{6 0 8 . 1 4 7}$ \\
Emi & 0.2196 & 0.22364 & 0.22015 & 0.22364 \\
\hline
\end{tabular}


Table 6: Best Solutions for Emission with Transmission Losses

\begin{tabular}{ccccc} 
& SDP & NPGA & SPEA & NSGA-II \\
\hline$P_{g_{1}}$ & 0.4097 & 0.3923 & 0.4043 & 0.4141 \\
$P_{g_{2}}$ & 0.4626 & 0.4700 & 0.4525 & 0.4602 \\
$P_{g_{3}}$ & 0.5424 & 0.5565 & 0.5525 & 0.5429 \\
$P_{g_{4}}$ & 0.3886 & 0.3695 & 0.4079 & 0.4011 \\
$P_{g_{5}}$ & 0.5425 & 0.5599 & 0.5468 & 0.5422 \\
$P_{g_{6}}$ & 0.5142 & 0.5163 & 0.5005 & 0.5045 \\
\hline Cost & 644.1105 & 645.984 & 642.603 & 644.133 \\
Emi & $\mathbf{0 . 1 9 4 1 8 3}$ & $\mathbf{0 . 1 9 4 2 4}$ & $\mathbf{0 . 1 9 4 2 2}$ & $\mathbf{0 . 1 9 4 1 9}$ \\
\hline
\end{tabular}




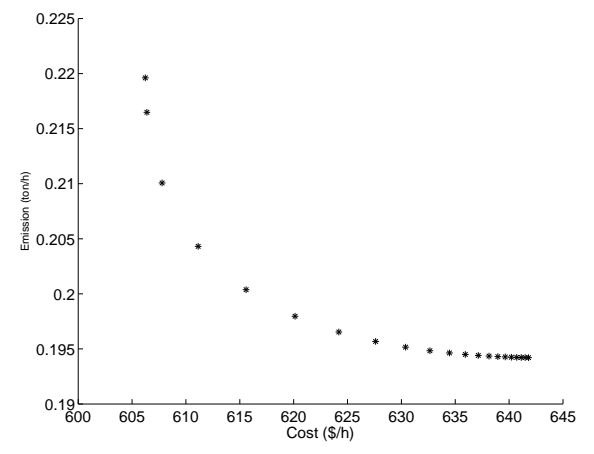

(a) $k_{1}=2$

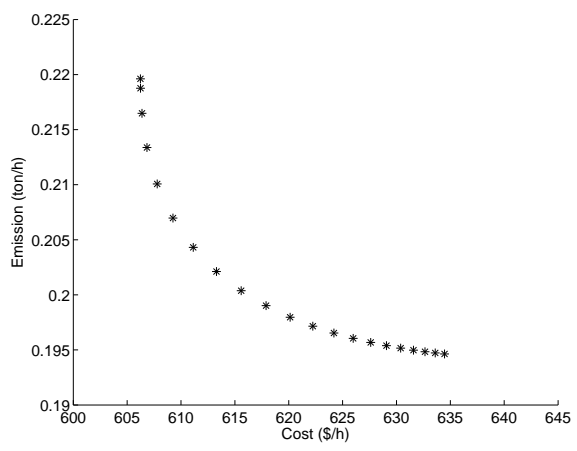

(b) $k_{1}=6$

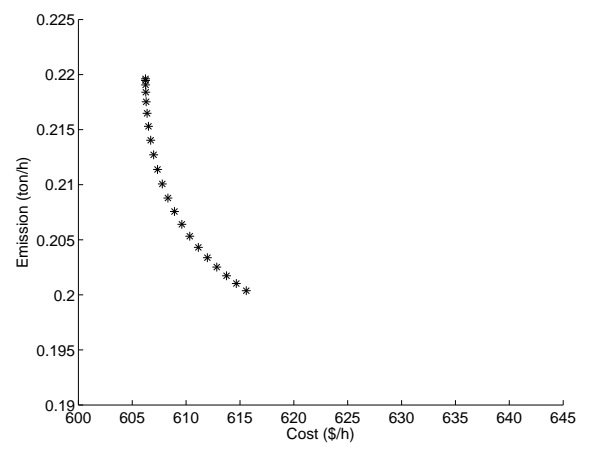

(c) $k_{1}=10$

Figure 4: Effect of variation of $k_{1}$ on the distribution of the Pareto front

Table 7: Summary of best solutions for the 6-unit test system

\begin{tabular}{lcccc}
\hline & \multicolumn{2}{c}{ Min. Fuel } & \multicolumn{2}{c}{ Min. Emission } \\
$P_{D}(M W)$ & Fuel $\cdot 10^{-3}$ & Emi $\cdot 10^{-3}$ & Fuel $\cdot 10^{-3}$ & Emi $\cdot 10^{-3}$ \\
\hline 150 & $\mathbf{2 . 6 9 8}$ & 8.137 & 2.7093 & $\mathbf{8 . 0 6 2}$ \\
175 & $\mathbf{3 . 1 6 8}$ & 10.008 & 3.205 & $\mathbf{9 . 7 1 5}$ \\
200 & $\mathbf{3 . 7 1 9}$ & 11.709 & 3.741 & $\mathbf{1 1 . 5 0 4}$ \\
225 & $\mathbf{4 . 3 6 8}$ & 13.131 & 4.371 & $\mathbf{1 3 . 0 7 0}$ \\
\hline
\end{tabular}


Table 8: Solution of the 6 -unit test bus with equally weighted objectives

\begin{tabular}{lcccccccc}
\hline \multicolumn{1}{c}{$P_{D}$} & \multicolumn{2}{c}{$150 \mathrm{MW}$} & \multicolumn{2}{c}{$175 \mathrm{MW}$} & \multicolumn{2}{c}{$200 \mathrm{MW}$} & \multicolumn{2}{c}{$225 \mathrm{MW}$} \\
\hline & SDP & LAN & SDP & LAN & SDP & LAN & SDP & LAN \\
\hline$P_{g_{1}}$ & 50.00 & 50.02 & 50.00 & 52.88 & 50.00 & 53.64 & 50.00 & 51.06 \\
$P_{g_{2}}$ & 20.00 & 21.06 & 20.00 & 21.98 & 30.09 & 22.45 & 45.69 & 24.90 \\
$P_{g_{3}}$ & 15.00 & 16.03 & 15.00 & 18.08 & 15.00 & 17.03 & 24.30 & 18.59 \\
$P_{g_{4}}$ & 21.57 & 16.95 & 30.96 & 28.02 & 35.00 & 40.99 & 35.00 & 49.67 \\
$P_{g_{5}}$ & 20.24 & 34.05 & 26.99 & 43.98 & 30.00 & 48.57 & 30.00 & 57.10 \\
$P_{g_{6}}$ & 23.19 & 12.23 & 32.04 & 12.04 & 39.91 & 19.45 & 40.00 & 28.17 \\
\hline Fuel $\cdot 10^{-3}$ & 2.700 & 2.789 & 3.171 & 3.350 & 3.720 & 3.934 & 4.369 & 4.569 \\
Emi $\cdot 10^{-3}$ & 8.105 & 8.142 & 9.951 & 10.08 & 11.71 & 12.19 & 13.09 & 14.54 \\
\hline
\end{tabular}

Table 9: The values of the aggregated objective function obtained by different methods for different values of the weight factors

\begin{tabular}{|c|c|c|c|c|c|c|c|c|c|c|c|}
\hline$w_{1}$ & 1.0 & 0.9 & 0.8 & 0.7 & 0.6 & 0.5 & 0.4 & 0.3 & 0.2 & 0.1 & 0.0 \\
\hline \multicolumn{12}{|l|}{ Algorithm } \\
\hline GA & 21.117 & 19.667 & 18.804 & 16.975 & 16.216 & 14.953 & 14.564 & 12.975 & 12.033 & 10.875 & 8.416 \\
\hline BFA & 21.244 & 20.668 & 18.269 & 16.982 & 16.113 & 14.839 & 13.463 & 12.723 & 10.916 & 9.105 & 8.170 \\
\hline PSO & 20.827 & 19.288 & 18.200 & 16.522 & 15.642 & 13.999 & 12.736 & 12.157 & 10.243 & 9.177 & 8.007 \\
\hline BFNM & 20.253 & 19.006 & 17.778 & 16.507 & 15.196 & 13.923 & 12.618 & 11.329 & 9.767 & 8.645 & 7.348 \\
\hline $\mathrm{POP} / \mathrm{SDP}$ & 19.740 & 18.517 & 17.256 & 15.991 & 14.724 & 13.456 & 12.188 & 10.921 & 9.653 & 8.385 & 7.117 \\
\hline
\end{tabular}

